JOURNAL OF SYNCHROTRON RADIATION

ISSN 1600-5775

Received 14 September 2021

Accepted 14 December 202

Edited by M. Yabashi, RIKEN SPring-8 Center, Japan

Keywords: serial crystallography; microfluidic; PROXIMA-1.

Supporting information: this article has supporting information at journals.iucr.org/s

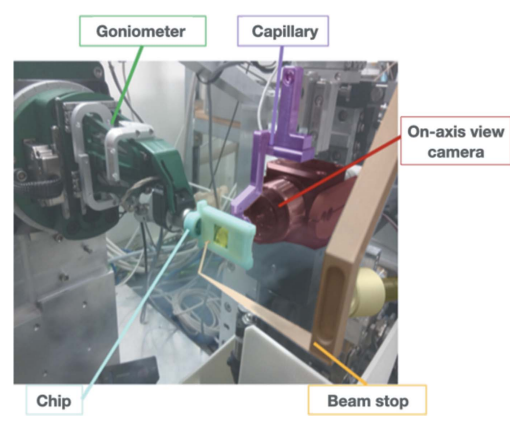

OPEN $\odot$ ACCESS

\section{Implementation of wedged-serial protein crystallography at PROXIMA-1}

\author{
Igor Chaussavoine, ${ }^{\mathrm{a}}$ Tatiana Isabet, ${ }^{\mathrm{a}}$ Robin Lener, ${ }^{\mathrm{a}}$ Pierre Montaville, ${ }^{\mathrm{a}}$ \\ Ramakrishna Vasireddi ${ }^{a}$ and Leonard M. G. Chavas ${ }^{b}$ *

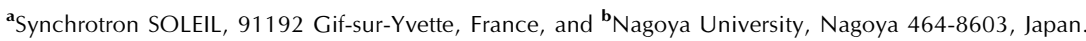 \\ *Correspondence e-mail: I.chavas@nusr.nagoya-u.ac.jp
}

An approach for serial crystallography experiments based on wedged-data collection is described. This is an alternative method for recording in situ X-ray diffraction data on crystalline samples efficiently loaded in an X-ray compatible microfluidic chip. Proper handling of the microfluidic chip places crystalline samples at geometrically known positions with respect to the focused X-ray interaction area for serial data collection of small wedges. The integration of this strategy takes advantage of the greatly modular sample environment available on the endstation, which allows access to both in situ and more classical cryocrystallography with minimum time loss. The method represents another optional data collection approach that adds up to the already large set of methods made available to users. Coupled with the advances in processing serial crystallography data, the wedged-data collection strategy proves highly efficient in minimizing the amount of required sample crystals for recording a complete dataset. From the advances in microfluidic technology presented here, highthroughput room-temperature crystallography experiments may become routine and should be easily extended to industrial use.

\section{Introduction}

Serial protein crystallography at synchrotrons has grown in interest following the developments at $\mathrm{X}$-ray free-electron laser (XFEL) sources for X-ray diffraction data collection (Grünbein \& Kovacs, 2019; Chavas et al., 2015) with reduced damages (Nass, 2019), a serious obstacle for sensitive biological samples. For the XFEL approach, in contrast to the more classical synchrotron cryo-crystallography, hundreds of thousands of in situ crystals are exposed to extremely intense $\mathrm{X}$-rays that lead to a unique diffraction image recorded per crystal using the principle referred to as 'diffraction before destruction' (Neutze et al., 2000; Johansson et al., 2017). The great need for, and consumption of, samples represent the main caveat of this XFEL approach, classically requiring between 100000 and several million crystal diffraction images to confidently solve a macromolecular structure (Chapman et al., 2011; Chapman \& Fromme, 2017). XFEL and synchrotron sources differ in the nature of the photons being distributed, which, in turn, affects the type of studies that can be performed, the overall instrumentation on the experimental station, how the sample should be prepared and handled, and how data analysis needs to be carried out. Instrumental developments, however, are often shared or adapted, especially when referring to sample preparation and handling. By taking advantage of technological improvements related to the speed of data recording, size and quality of X-ray beams, identification and handling of smaller samples, and data processing methods, strong interest has grown at synchrotron 
Table 1

Major sample (protein crystals) delivery systems used at X-ray sources.

This comparative summary is inspired by Lyubimov et al. (2015) and represents various existing liquid-jet and fixed-target sample delivery methods notably used for serial femtosecond crystallography experiments at XFEL sources. The Efficiency column represents an estimation of the average number of crystals per integrated image based on the information provided in the reference.

\begin{tabular}{|c|c|c|c|}
\hline Method & Efficiency & Advantages & Limitations \\
\hline GDVN jet & 25000 (Sierra et al., 2012) & Low background, crystals in crystallization buffer & $\begin{array}{l}\text { High sample consumption, possible clogging } \\
\text { of the system, possible sample damage }\end{array}$ \\
\hline Electrospinning jet & 500 (Sierra et al., 2012) & Low background, low flow rate & $\begin{array}{l}\text { Require viscous media, potential impact of } \\
\text { electrostatic charge on samples }\end{array}$ \\
\hline LCP jet & 500 (Weierstall et al., 2014) & Low background, low flow rate, lipidic cubic phase & Crystals must grow in lipidic cubic phase \\
\hline Micromesh & Not determined & $\begin{array}{l}\text { Rapid data collection, small sample size, fits standard } \\
\text { goniometer }\end{array}$ & $\begin{array}{l}\text { Freezing and cryopreservation required, need } \\
\text { multiple devices for complete datasets }\end{array}$ \\
\hline Levitation droplets & 2 (Roessler et al., 2016) & Precise sample delivery, crystals in crystallization buffer & Solvent background scattering, evaporation \\
\hline Silicon chips & 11 (Roedig et al., 2017) & Precise sample delivery, crystals in crystallization buffer & Evaporation \\
\hline
\end{tabular}

facilities for employing a variant of XFEL serial crystallography as a more standardized method for protein crystal X-ray diffraction (Pearson \& Mehrabi, 2020; Martin-Garcia, 2021; Diederichs \& Wang, 2017; Owen et al., 2017).

A variety of strategies to study crystals at synchrotron and room temperature has led to the engineering of approaches that can be divided into moving-target and fixed-target systems (a non-exhaustive list of strategies is reported Table 1). The concept of imaging X-ray damage-free structures while applying intense beams at third-generation synchrotrons was first explored using capillaries and in-flow sample delivery (Stellato et al., 2014). Rather than capillaries, microfluidic chips were also interfaced for diffraction measurements using a combination of continuous crystal flow and small-wedged rotation (Monteiro et al., 2020). The implementation of highviscosity extrusion injectors represents yet another example of moving targets (Botha et al., 2015), with a real advantage of the technique when considering time-resolved experiments. The above examples efficiently replenish samples, and generally do not require external instrumentation; however, the position and orientation of the samples remain unknown which renders the data processing somewhat complicated.

Taking advantage of automation and state-of-the-art goniometry implemented at macromolecular crystallography beamlines, great efforts are provided in developing data collection strategies from fixed-target systems. The lead innovation flag behind these sample-delivery techniques targets a reduction in sample consumption. The conveyor belts approach (Soares et al., 2014) returned data comparable to mesh-loops with a great potential for reducing the solvent around the crystals for an improved signal-to-noise ratio, although aggregation of crystals was reported and may affect data quality. The use of patterned chips represents most of the recent engineering observed by various groups. Among the various methods already implemented at synchrotrons, Roedig et al. (2016, 2017) presented a silicon-based handling chip optimized for high-speed data collection of crystal samples presented on a fixed target, at both synchrotron and XFEL facilities. The preparation protocol of the chip and its nature induced a stochastic orientation and positioning of the crystals, which lead to hit rates at XFELs of $2 \%$ to $10 \%$, yet representing a comfortable ratio for 'placed crystal' over 'diffracted crystal' higher when compared with other sampledelivery approaches.

In order to improve data quality and open the field to a larger set of samples, we have chosen to concentrate on yet another method for crystal delivery, based on handling crystal samples by microfluidic devices. In previous studies, Lyubimov et al. (2015) introduced a technique that reduces sample consumption by placing the crystals to be diffracted at known positions within a microfluidic chip. The technique drastically reduces crystal usage while preventing dehydration of samples and performing the experiments at room temperature. The device can be adapted to either XFEL or synchrotron experiments; however, specific hardware adaptations are required to operate the chips in the sample environment. One of the biggest advantages in experimenting with these devices lies in the possibility to perform multi-crystal diffraction data collection, which greatly reduces the impact of radiation damage to the sample (Gotthard et al., 2019). Most surprisingly, the technique introduced by Lyubimov and coworkers has not been reported as extensively used, although it represents an optimized solution for fast and assisted positioning of the crystals at the synchrotron X-ray beam interaction point.

In line with the goal of minimizing sample loss, the microfluidic technology is, in essence, based on very low consumption of injected materials. When applied to macromolecular crystallography, microfluidic chips have to be compatible with the diffraction experiments, often framed by thin films composed of elements that weakly absorb X-rays. Depending on the design of the chip, the loading of samples may be delicate and eventually become a challenging process. Additionally, the highly random distribution of crystals within the microfluidic chips complicates data collection, classically performed without considering the individual positions of the crystals and exposing them to the X-ray beam in an unsystematic manner.

In the current work, a simplified design of the Lyubimov et al. (2015) approach was engineered to isolate crystalline samples at positions geometrically known within the microfluidic device. Using the chip as a sample holder for tensto-hundreds of crystals, full datasets on test samples were recorded at room temperature with data collection performed over a small rotation angle for each crystal position, with low- 
dose exposition and continuous rotation for an easier indexing of the reflections. During each data collection, the microfluidic chip is not translated, and the crystals are fully centered at the $\mathrm{X}$-ray interaction area while being rotated. Owing to the low X-ray absorption of the chip, the data collection method demonstrates efficient in situ diffraction collection with low sample consumption. Additionally, the small physical dimensions of the device minimize the risk of hardware collision with the beamline equipment while recording larger rotation angles. Taken together with its applicability to diffraction experiments for macromolecular crystallography, this opens the possibility of implementing the chip at most synchrotron MX beamlines equipped with a goniometer head accepting magnet-type sample holders.

\section{Material and methods}

\subsection{Sample preparation}

Lysozyme crystals were prepared in batches to generate a range of sizes with optimized dimensions of $15 \mu \mathrm{m}$ for the longest direction. Lysozyme powder (Sigma-Aldrich) was dissolved in a buffer of $50 \mathrm{~m} M$ acetate mixed with the mother liquor solution $(1 \mathrm{M} \mathrm{NaCl}, 35 \%$ ethylene glycol, $12.5 \%$ PEG3350, $50 \mathrm{~m} M$ acetate), incubated for $15 \mathrm{~h}$ at $283 \mathrm{~K}$ before storage at $253 \mathrm{~K}$.

Insulin crystals were produced from a powder (SigmaAldrich) dissolved in $10 \mathrm{mM} \mathrm{HCl}, 50 \mathrm{~m} M$ citrate and $6 \mathrm{mM}$ zinc sulfate and pre-incubated at $323 \mathrm{~K}$ for $20 \mathrm{~min}$. The solution was then supplemented by $15 \%$ acetone, incubated for an additional $20 \mathrm{~min}$ at $323 \mathrm{~K}$ and the crystallization was quenched by an incubation at $293 \mathrm{~K}$ for $6 \mathrm{~h}$ before storage at $277 \mathrm{~K}$.

\subsection{Microfluidic chip manufacturing}

The chip was manufactured in a stepwise process using standard methods of spin-coating and plasma bonding (Fig. 1). The trapping pattern was UV-insulated on the $40 \mu \mathrm{m}$-thick spin-coated resin (SU-8 2015) using a laser writer (KLOE). After development of the resin and PDMS molding, holes for the inlets and outlets were punched and a final step of plasma bonding on a $25 \mu \mathrm{m}$ Kapton support hermetically closed the chip. The design of the chip was optimized to trap crystals measuring $15 \mu \mathrm{m}$ to $50 \mu \mathrm{m}$ (longest direction). The trap channel width is $10 \mu \mathrm{m}$, leading to difficulty in trapping samples smaller than $15 \mu \mathrm{m}$. With the current design, about $20 \%$ of the manufactured chips were deficient while presenting leaks when injecting buffers, most likely caused by operational fluctuations of the equipment employed to make the chips.

\subsection{Sample injection}

Great care was taken while injecting the samples inside the chip, to avoid unnecessary mechanical stress on the crystals and favor efficient trapping. The most reproducible injection protocol results from the use of a pressure controller (Fluigent) for the injection of the crystal-containing solution into plastic tubing of $0.5 \mathrm{~mm}$ inner diameter. For an efficient
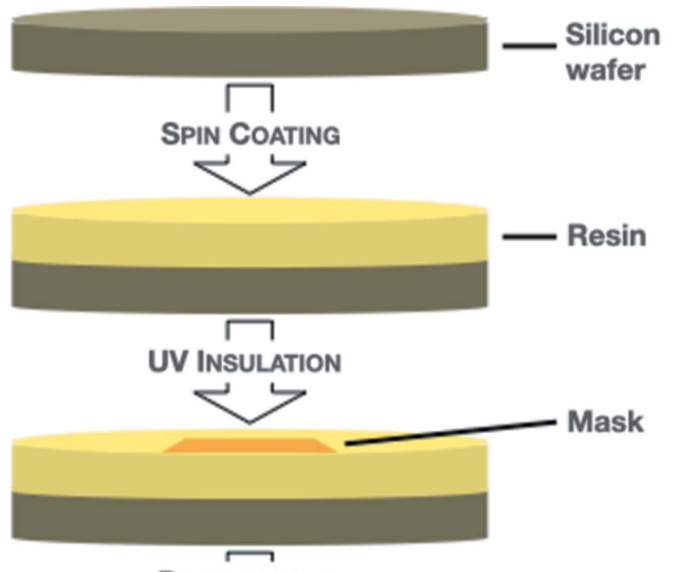

DeVELOPMENT

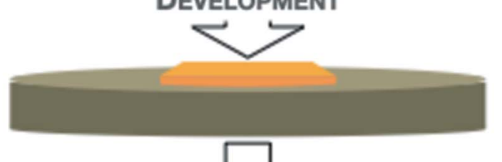

$\square$

MOLDING

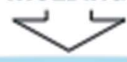

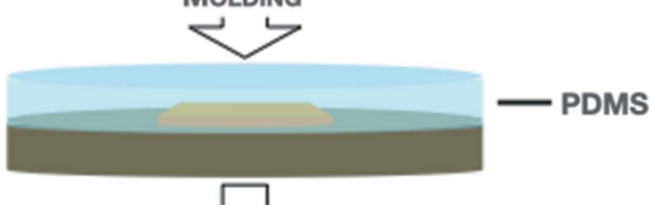

Peeling OfF

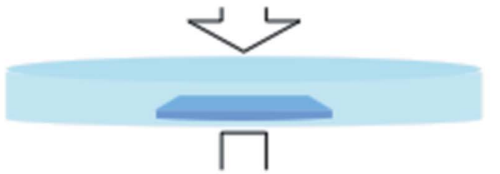

PUNCHING

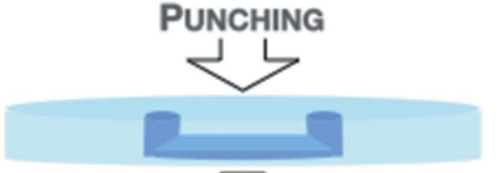

$\square$

PLASMA BONDING

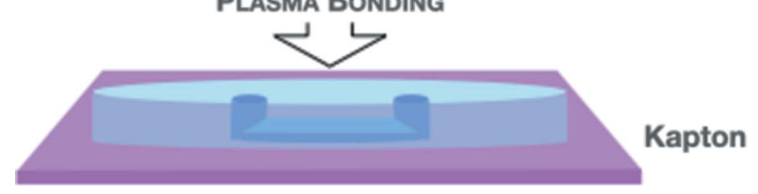

Figure 1

Scheme of the various steps involved in manufacturing the chip.

loading, the sample-free crystal solution was first injected with a pressure of $50 \mathrm{mBar}$ for long periods $(10 \mathrm{~h})$ to remove all potential air bubbles within the channels (see Fig. S1 and Movie S1 of the supporting information). When ready, the chip was then loaded with the samples after gentle mixing to minimize sedimentation of the crystals. Before mounting on the beamline goniometer head, the chip was inspected for the presence of crystals in the traps under microscopes.

\subsection{Chip handling}

Handling of the microfluidic chip was performed at the PROXIMA-1 beamline of Synchrotron SOLEIL, France (Chavas et al., 2021). The final PDMS chip loaded with crystals is $20 \mathrm{~mm}$ in width and $30 \mathrm{~mm}$ in height. It is accommodated 
inside an adapted 3D-printed frame for further mounting on a magnet-type goniometer head (Fig. S2). Though with a different design, use of a 3D-printed frame has already been reported (Broecker et al., 2018; Huang et al., 2020) and presents the advantage to rigidify the chip, which allows easier handling and eventually robot-assisted mounting. Additionally, and most importantly, using such a tool reduces the physical stress and, in turn, avoids liquid/sample movements inside the chip during manipulation. Based on the geometry of the frame, the sample environment at the beamline was adapted to avoid any potential collision of hardware with the rotating chip.

\subsection{In situ geometrically optimized raster scanning}

The microfluidic chips were designed to place the samples at known spatial positions to optimize in situ data collection at each position without spending time to re-center. In situ geometrically optimized raster (IGOR) scanning was implemented within an optional package of the $M X C u B E$ GUI (Gabadinho et al., 2010) specifically designed for this purpose (code available on request). In brief, the chip mounted on the goniometer head is pre-centered on three fiducials, usually represented by crystal positions marked and understood within $M X C u B E$ as being the alignment points for the chip. Based on the recorded positions of these three marks, $M X C U B E$ can calculate the coordinates for all the traps within the chip and uses these coordinates to automatically and sequentially position all the crystals at the X-ray interaction area. Wedges of a few degrees are then recorded at each position.

\subsection{Data processing, analysis and structure determination}

All data collections were performed at PROXIMA-1 (Chavas et al., 2021). Data on lysozyme crystals were collected at room temperature $(\sim 294 \mathrm{~K})$ on a single row of 30 crystals by recording wedges of $30^{\circ}$ per crystal, with oscillations of $0.1^{\circ}$ and an exposure time of $0.10 \mathrm{~s}$ on a Pilatus-6M (Dectris, Ltd). To avoid strong damages to the in situ crystals, the X-ray full beam at $12.67 \mathrm{keV}$ energy $\left(\sim 2 \times 10^{11}\right.$ photons $\left.\mathrm{s}^{-1}\right)$ was attenuated to $\sim 5 \times 10^{9}$ photons $\mathrm{s}^{-1}$, which corresponds to a dose of approximately $58 \mathrm{kGy}$ per crystal, below the recommended dose for in situ data collection (de la Mora et al., 2020). Data on insulin crystals were collected using the IGOR scanning procedure by recording wedges of $10^{\circ}$ with $0.05^{\circ}$ oscillations at a frequency of $20 \mathrm{~Hz}$, on an EigerX-16M (Dectris) at $12.67 \mathrm{keV}$ energy and for an attenuated X-ray beam of $\sim 5 \times 10^{9}$ photons s $^{-1}$.

Data were processed with XDS (Kabsch, 2010) through the autoProc package (Vonrhein et al., 2011). Datasets were converted to MTZ format by POINTLESS (Evans, 2005), and scaled and merged by AIMLESS (Evans \& Murshudov, 2013), as implemented within the autoProc procedure, taking $C C_{1 / 2} \geq 0.85$ and $I / \operatorname{sig}(I) \geq 2.5$ as the resolution cutoff criteria in the outermost resolution shell. Calculations for dose deposition were performed using RADDOSE-3D (Zeldin et al., 2013).
Table 2

Data collection statistics.

Values in parentheses correspond to the highest resolution shell.

\begin{tabular}{lll}
\hline Data collection & Lysozyme & Insulin \\
\hline Number of merged data & 30 & 13 \\
Space group & $P 4_{3} 2_{1} 2$ & $R 3$ \\
Unit-cell parameters $(\AA)$ & $a=b=79.67, c=37.90$ & $a=b=83.08, c=34.39$ \\
Resolution $(\AA)$ & $35.63-1.60(1.64-1.60)$ & $41.54-2.33(2.39-2.33)$ \\
No. of observed reflections & $116929(5636)$ & $9615(168)$ \\
No. of unique reflections & $16516(1196)$ & $2464(102)$ \\
Completeness $(\%)$ & $99.3(99.3)$ & $65.1(37.0)$ \\
Multiplicity & $7.1(4.7)$ & $3.9(1.6)$ \\
$R_{\text {merge }}$ & $0.064(0.891)$ & $0.295(0.554)$ \\
$C C_{1 / 2}$ & $0.997(0.658)$ & $0.890(0.560)$ \\
\hline
\end{tabular}

Structure-factor amplitudes were obtained by TRUNCATE (French \& Wilson, 1978). Rotational and translational functions were calculated and compared by MOLREP (Vagin \& Teplyakov, 2010) using the coordinates of the PDB entries 4dt3 (Cha et al., 2012) and 3w7z (Hoshikawa et al., unpublished) as template models for lysozyme and insulin, respectively. The solved structures were then run through rounds of refinement with BUSTER (Bricogne et al., 2017), and manual model buildings using Coot (Emsley et al., 2010). Data analysis and refinement statistics are shown in Table 2.

\section{Results and discussion}

In the course of this work, the microfluidic chips developed for the IGOR scanning have been subject to constant improvement. Among the numerous versions of the device, efforts were made to both decrease the thickness of the chip for improving its response to X-ray exposition and optimize the geometry of the channels to decrease the risk of clogging while increasing the probability of trapping (Fig. 2). In an optimized version, the chip is made of 180 independent traps arranged in three double-rows; this block element can be multiplied to scale up the number of traps; however, the injection protocol may need to be adapted. Both the geometry of the channels and the thickness of the chip ( $100 \mu \mathrm{m}$ in the beam direction) are optimized for crystals of dimensions ranging from $15 \mu \mathrm{m}$ to $50 \mu \mathrm{m}$ in the longest direction.

In order to acquire high-quality X-ray diffraction data from the trapped samples, the chip has to be centered to the X-ray interaction point with micrometer precision using the available goniometer. The reduced thickness of the chip makes it highly bendable and therefore increases the need for a stiff handling structure, which takes the form of an interface between the chip and the goniometer to ensure effective manipulation. Such a procedure was used and reported previously for other in situ applications (Broecker et al., 2018; Huang et al., 2020). In the current work, a specific 3D-printed encapsulating frame was designed to give a rigid support that protects the chip from mechanical stress and deformation and permits the chip to be gripped and lifted either manually or by a sample-mounting robot (Fig. S2). The addition of a magnetic cap allows accommodation on the conventional goniometer head (Fig. 3). 


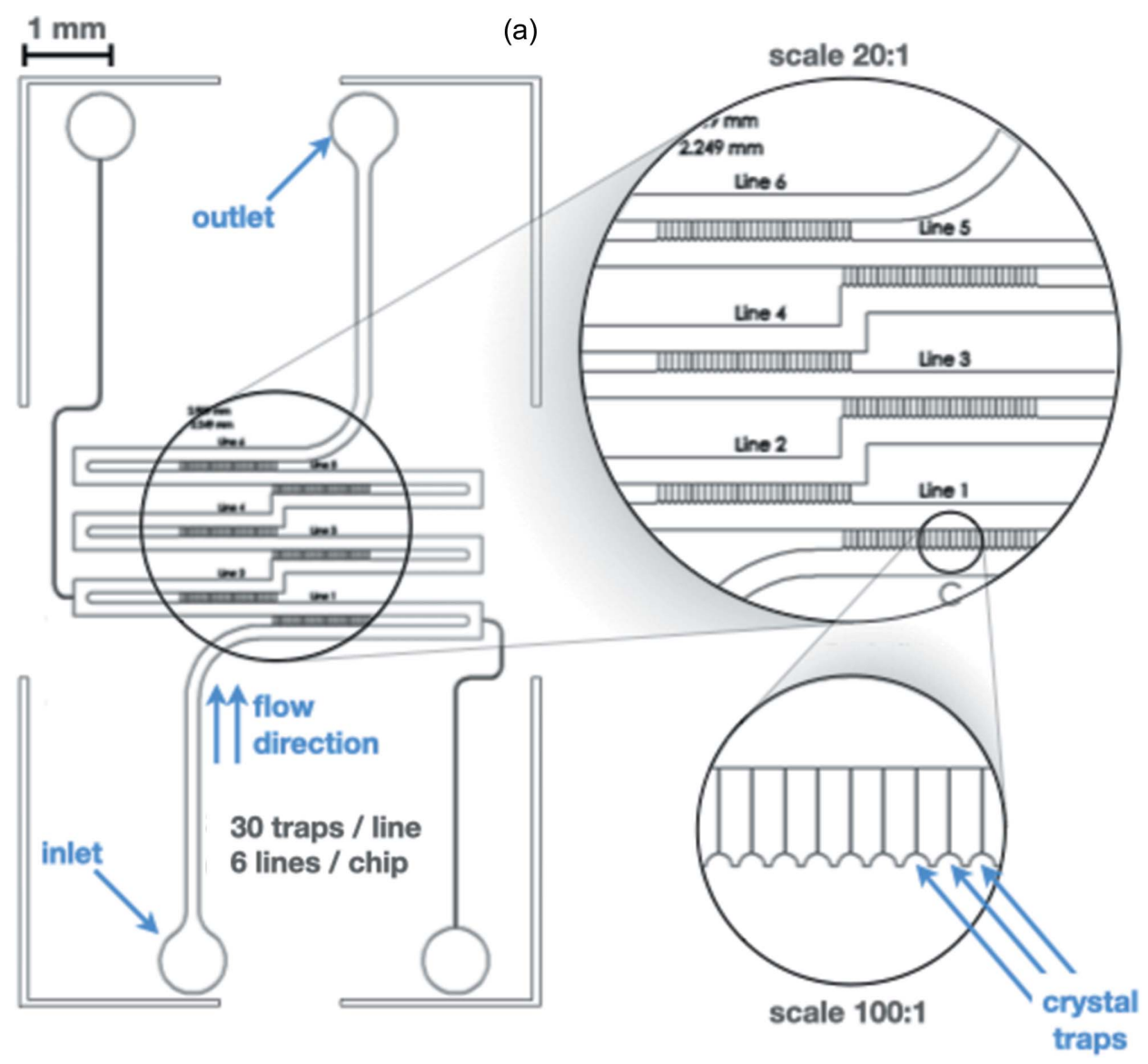

(b)
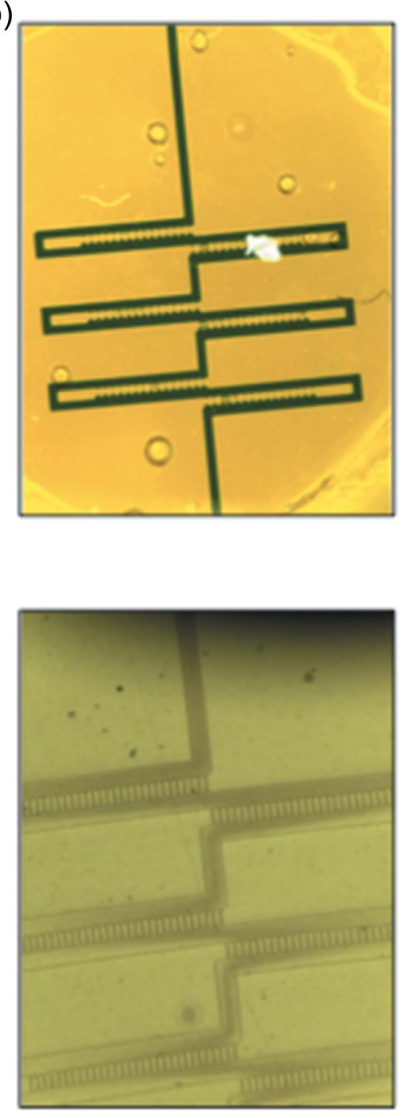

Figure 2

Design of the trapping chip. (a) CAD-drawing of the chip, highlighting the arrangement of 30 traps within each of the 6 lines contained in the device. Inlet, outlet, flow direction and trap locations are indicated in blue. (b) Images of two trapping chips loaded with different colorant to increase the contrast and highlight the design.

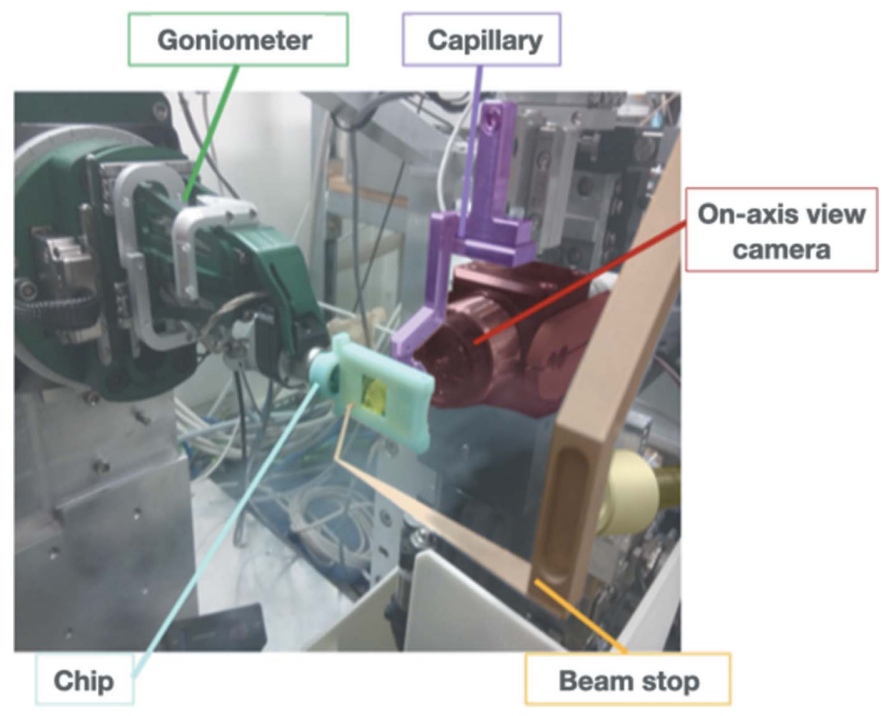

Figure 3

PROXIMA-1 beamline sample environment with a microfluidic chip mounted on the goniometer. The capillary and the beam stop are inserted with the cryostream retracted, the beamline is configured for data collection and the microfluidic chip is encapsulated within a 3D-printed frame to assist handling.
Centering and handling of both the microfluidic chip mounted on the goniometer and the crystals within is performed through the $M X C U B E$ user interface. To facilitate centering of all the traps with the minimum actions, the original version of $M X C U B E$ has been modified with options where the chips can be handled and IGOR scans can be scheduled (the details of these modifications will be described elsewhere). As a result of a 3-point centering operation, all the traps are recognized and marked as potential positions for further data collection. The users then have the possibility to either select all the positions or only those at which data collection should be preferentially performed.

The IGOR scanning method applies a simplified wedgedserial crystallography strategy of collecting X-ray diffraction data. Such a small-wedged data collection strategy was used early in the structural studies of sensitive membrane proteins (Cherezov et al., 2007), and was recently successfully implemented within automated data collection pipelines at the ESRF and SPring-8 facilities (Zander et al., 2015; Hirata et al., 2019). In the current procedure, after moving the crystal to the center of the X-ray beam, fine-slice wedges of few degrees $\left( \pm 10^{\circ}\right.$ by default) are collected before moving to the next target [Fig. 4(a)]. Contrary to previously reported serial synchrotron rotation crystallography strategies (Gati et al., 
2014; Hasegawa et al., 2017; Roedig et al., 2017), the X-ray beam fast-shutter is closed during the centering of the crystals and the goniometer position is oriented back to its starting
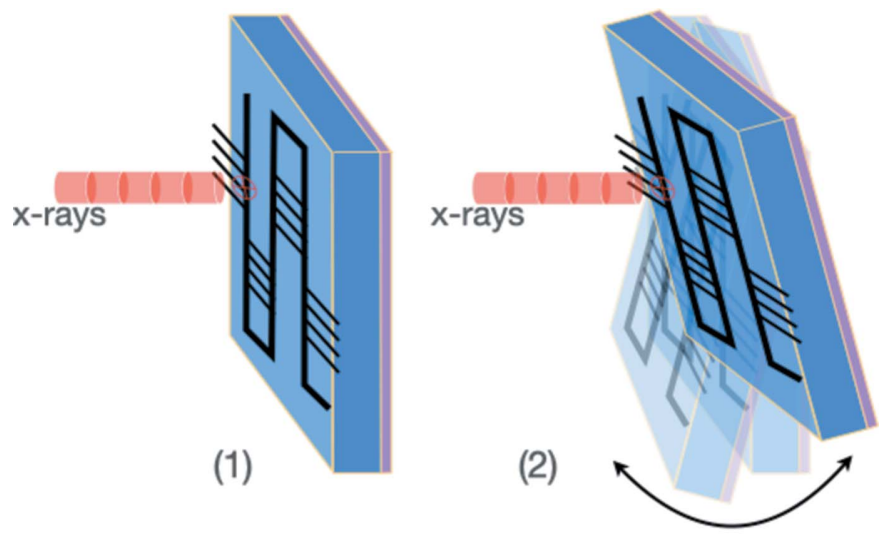

$\pm 10^{\circ}$

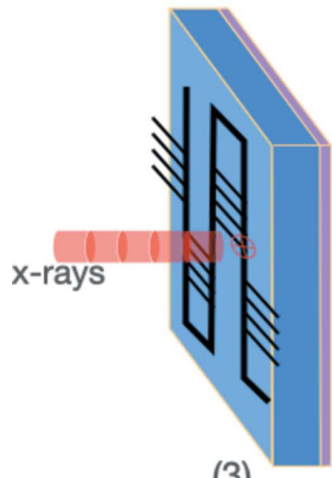

(3)

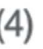

(a)

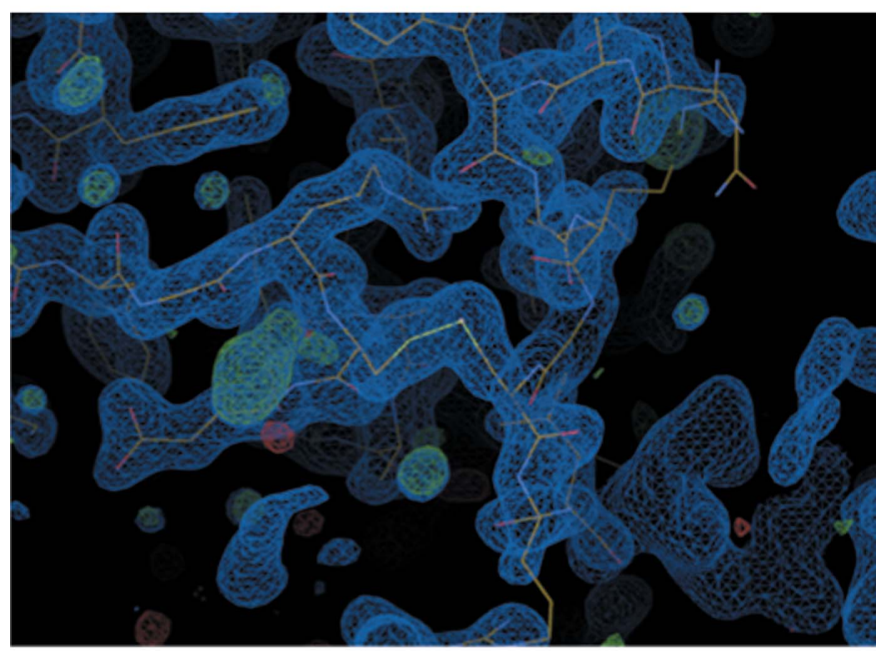

(b)

Figure 4

Data collection protocol and resulting electron density from the lysozyme crystal structure. (a) Data collection strategy using small wedges on microfluidic chips. (1) The first crystal position is brought to the beam, (2) followed by data collection of $\pm 10^{\circ}$ around the centered position. (3) The chip is then moved to another position and (4) data collection is repeated. (b) Electron density of the lysozyme structure obtained after structure determination and with no round of refinement. angle before each X-ray exposure. Data collection on all crystals within the chip takes consequently longer; however, the choice was made to minimize the number of photons delivered on the crystals to reduce unnecessary radiation damage while keeping the benefits of collecting continuous oscillation data.

Data processing of the serial crystallography data was performed manually, and data were selected for merging based on identical crystal lattice parameters. Overall, 9 out of 30 data were selected for lysozyme crystals, whereas many more data (a full chip or 180 traps) were collected for insulin for only 13 that could be merged. Phasing of lysozyme and insulin structures was performed with the sole purpose of confirming that no major issues occur within the protein structures; thus, a single run of restrained refinement followed the molecular replacement steps, with no additional rebuilding of sides chains nor inclusion of water molecules. The structures show no additional feature when compared with already well documented structures, with radiation damages clearly not visible from the electron density maps at these resolutions [Fig. 4(b)].

\section{Conclusions}

The exciting progress witnessed in the development of microfluidic chips applied to biological objects illustrates the great potential of these devices for handling samples that are classically difficult to manipulate, fragile or even hazardous. Operation of the microfluidic chips remains delicate, and great care should be provided when exposing the crystals to strong $\mathrm{X}$-rays at room temperature. Additionally, the nature and choice of the compounds used to manufacture the chip will strongly affect the absorption of the incoming X-rays and resulting recorded noise, which will directly impair the quality of the recorded data. The current work introduces a lowabsorption device; however, improvements should be provided to further minimize the background noise originating from the chip. The IGOR scanning approach appears as an effective solution to the merging of serial X-ray diffraction data, with indexing facilitated by an automated pre-centering of the crystals trapped in the chip and through recording continuous oscillation angles. This approach will remain of greater interest for samples with data classically difficult to index, weak to radiation damages or for which careful data collection protocols should be applied.

\section{Acknowledgements}

The authors would like to thank the members of the microfluidic laboratory for device fabrication, the staff of the PROXIMA-1 beamline at Synchrotron SOLEIL and all associated scientists that helped in the data collection and analysis carried out during commissioning beam time. The complete set of raw diffraction images, associated processed data and specific $M X C U B E$ codes related to the IGOR scans are available upon request from the authors. 


\section{References}

Botha, S., Nass, K., Barends, T. R. M., Kabsch, W., Latz, B., Dworkowski, F., Foucar, L., Panepucci, E., Wang, M., Shoeman, R. L., Schlichting, I. \& Doak, R. B. (2015). Acta Cryst. D71, 387397.

Bricogne, G., Blanc, E., Brandl, M., Flensburg, C., Keller, P., Paciorek, W., Roversi, P., Sharff, A., Smart, O. S., Vonrhein, C. \& Womack, T. O. (2017). BUSTER, version 2.10.2. Cambridge, UK: Global Phasing Ltd.

Broecker, J., Morizumi, T., Ou, W.-L., Klingel, V., Kuo, A., Kissick, D. J., Ishchenko, A., Lee, M.-Y., Xu, S., Makarov, O., Cherezov, V., Ogata, C. M. \& Ernst, O. P. (2018). Nat. Protoc. 13, 260-292.

Cha, S. S., An, Y. J., Jeong, C. S., Kim, M. K., Lee, S. G., Lee, K. H., Oh, B. H. (2012) Acta Cryst. D68, 1253-1258.

Chapman, H. N. \& Fromme, P. (2017). Curr. Opin. Struct. Biol. 45, 170-177.

Chapman, H. N., Fromme, P., Barty, A., White, T. A., Kirian, R. A., Aquila, A., Hunter, M. S., Schulz, J., DePonte, D. P., Weierstall, U., Doak, R. B., Maia, F. R., Martin, A. V., Schlichting, I., Lomb, L., Coppola, N., Shoeman, R. L., Epp, S. W., Hartmann, R., Rolles, D., Rudenko, A., Foucar, L., Kimmel, N., Weidenspointner, G., Holl, P., Liang, M., Barthelmess, M., Caleman, C., Boutet, S., Bogan, M. J., Krzywinski, J., Bostedt, C., Bajt, S., Gumprecht, L., Rudek, B., Erk, B., Schmidt, C., Hömke, A., Reich, C., Pietschner, D., Strüder, L., Hauser, G., Gorke, H., Ullrich, J., Herrmann, S., Schaller, G., Schopper, F., Soltau, H., Kühnel, K. U., Messerschmidt, M., Bozek, J. D., Hau-Riege, S. P., Frank, M., Hampton, C. Y., Sierra, R. G., Starodub, D., Williams, G. J., Hajdu, J., Timneanu, N., Seibert, M. M., Andreasson, J., Rocker, A., Jönsson, O., Svenda, M., Stern, S., Nass, K., Andritschke, R., Schröter, C. D., Krasniqi, F., Bott, M., Schmidt, K. E., Wang, X., Grotjohann, I., Holton, J. M., Barends, T. R., Neutze, R., Marchesini, S., Fromme, R., Schorb, S., Rupp, D., Adolph, M., Gorkhover, T., Andersson, I., Hirsemann, H., Potdevin, G., Graafsma, H., Nilsson, B. \& Spence, J. C. (2011). Nature, 470, 73-77.

Chavas, L. M. G., Gourhant, P., Guimaraes, B. G., Isabet, T., Legrand, P., Lener, R., Montaville, P., Sirigu, S. \& Thompson, A. (2021). J. Synchrotron Rad. 28, 970-976.

Chavas, L. M. G., Gumprecht, L. \& Chapman, H. N. (2015). Struct. Dyn. 2, 041709.

Cherezov, V., Rosenbaum, D. M., Hanson, M. A., Rasmussen, S. G. F., Thian, F. S., Kobilka, T. S., Choi, H.-J., Kuhn, P., Weis, W. I., Kobilka, B. K. \& Stevens, R. C. (2007). Science, 318, 1258-1265.

Diederichs, K. \& Wang, M. (2017). Methods Mol. Biol. 1607, 239-272.

Emsley, P., Lohkamp, B., Scott, W. G. \& Cowtan, K. (2010). Acta Cryst. D66, 486-501.

Evans, P. (2005). Acta Cryst. D62, 72-82.

Evans, P. R. \& Murshudov, G. N. (2013). Acta Cryst. D69, 1204-1214. French, S. \& Wilson, K. (1978). Acta Cryst. A34, 517-525.

Gabadinho, J., Beteva, A., Guijarro, M., Rey-Bakaikoa, V., Spruce, D., Bowler, M. W., Brockhauser, S., Flot, D., Gordon, E. J., Hall, D. R., Lavault, B., McCarthy, A. A., McCarthy, J., Mitchell, E., Monaco, S., Mueller-Dieckmann, C., Nurizzo, D., Ravelli, R. B. G., Thibault, X., Walsh, M. A., Leonard, G. A. \& McSweeney, S. M. (2010). J. Synchrotron Rad. 17, 700-707.

Gati, C., Bourenkov, G., Klinge, M., Rehders, D., Stellato, F., Oberthür, D., Yefanov, O., Sommer, B. P., Mogk, S., Duszenko, M., Betzel, C., Schneider, T. R., Chapman, H. N. \& Redecke, L. (2014). IUCrJ, 1, 87-94.

Gotthard, G., Aumonier, S., De Sanctis, D., Leonard, G., von Stetten, D. \& Royant, A. (2019). IUCrJ, 6, 665-680.

Grünbein, M. L. \& Nass Kovacs, G. (2019). Acta Cryst. D75, 178-191.

Hasegawa, K., Yamashita, K., Murai, T., Nuemket, N., Hirata, K., Ueno, G., Ago, H., Nakatsu, T., Kumasaka, T. \& Yamamoto, M. (2017). J. Synchrotron Rad. 24, 29-41.

Hirata, K., Yamashita, K., Ueno, G., Kawano, Y., Hasegawa, K., Kumasaka, T. \& Yamamoto, M. (2019). Acta Cryst. D75, 138-150.
Huang, C.-Y., Meier, N., Caffrey, M., Wang, M. \& Olieric, V. (2020). J. Appl. Cryst. 53, 854-859.

Johansson, L. C., Stauch, B., Ishchenko, A. \& Cherezov, V. (2017). Trends Biochem. Sci. 42, 749-762.

Kabsch, W. (2010). Acta Cryst. D66, 125-132.

Lyubimov, A. Y., Murray, T. D., Koehl, A., Araci, I. E., Uervirojnangkoorn, M., Zeldin, O. B., Cohen, A. E., Soltis, S. M., Baxter, E. L., Brewster, A. S., Sauter, N. K., Brunger, A. T. \& Berger, J. M. (2015). Acta Cryst. D71, 928-940.

Martin-Garcia, J. M. (2021). Crystals, 11, 521.

Monteiro, D. C. F., von Stetten, D., Stohrer, C., Sans, M., Pearson, A. R., Santoni, G., van der Linden, P. \& Trebbin, M. (2020). IUCrJ, 7, 207-219.

Mora, E. de la, Coquelle, N., Bury, C. S., Rosenthal, M., Holton, J. M., Carmichael, I., Garman, E. F., Burghammer, M., Colletier, J.-P. \& Weik, M. (2020). Proc. Natl Acad. Sci. 117, 4142-4151.

Nass, K. (2019). Acta Cryst. D75, 211-218.

Neutze, R., Wouts, R., van der Spoel, D., Weckert, E. \& Hajdu, J. (2000). Nature, 406, 752-757.

Owen, R. L., Axford, D., Sherrell, D. A., Kuo, A., Ernst, O. P., Schulz, E. C., Miller, R. J. D. \& Mueller-Werkmeister, H. M. (2017). Acta Cryst. D73, 373-378.

Pearson, A. R. \& Mehrabi, P. (2020). Curr. Opin. Struct. Biol. 65, 168174.

Roedig, P., Duman, R., Sanchez-Weatherby, J., Vartiainen, I., Burkhardt, A., Warmer, M., David, C., Wagner, A. \& Meents, A. (2016). J. Appl. Cryst. 49, 968-975.

Roedig, P., Ginn, H. M., Pakendorf, T., Sutton, G., Harlos, K., Walter, T. S., Meyer, J., Fischer, P., Duman, R., Vartiainen, I., Reime, B., Warmer, M., Brewster, A. S., Young, I. D., Michels-Clark, T., Sauter, N. K., Kotecha, A., Kelly, J., Rowlands, D. J., Sikorsky, M., Nelson, S., Damiani, D. S., Alonso-Mori, R., Ren, J., Fry, E. E., David, C., Stuart, D. I., Wagner, A. \& Meents, A. (2017). Nat. Methods, 14, 805-810.

Roessler, C. G., Agarwal, R., Allaire, M., Alonso-Mori, R., Andi, B., Bachega, J. F. R., Bommer, M., Brewster, A. S., Browne, M. C., Chatterjee, R., Cho, E., Cohen, A. E., Cowan, M., Datwani, S., Davidson, V. L., Defever, J., Eaton, B., Ellson, R., Feng, Y., Ghislain, L. P., Glownia, J. M., Han, G., Hattne, J., Hellmich, J., Héroux, A., Ibrahim, M., Kern, J., Kuczewski, A., Lemke, H. T., Liu, P., Majlof, L., McClintock, W. M., Myers, S., Nelsen, S., Olechno, J., Orville, A. M., Sauter, N. K., Soares, A. S., Soltis, S. M., Song, H., Stearns, R. G., Tran, R., Tsai, Y., Uervirojnangkoorn, M., Wilmot, C. M., Yachandra, V., Yano, J., Yukl, E. T., Zhu, D. \& Zouni, A. (2016). Structure, 24, 631-640.

Sierra, R. G., Laksmono, H., Kern, J., Tran, R., Hattne, J., AlonsoMori, R., Lassalle-Kaiser, B., Glöckner, C., Hellmich, J., Schafer, D. W., Echols, N., Gildea, R. J., Grosse-Kunstleve, R. W., Sellberg, J., McQueen, T. A., Fry, A. R., Messerschmidt, M. M., Miahnahri, A., Seibert, M. M., Hampton, C. Y., Starodub, D., Loh, N. D., Sokaras, D., Weng, T.-C., Zwart, P. H., Glatzel, P., Milathianaki, D., White, W. E., Adams, P. D., Williams, G. J., Boutet, S., Zouni, A., Messinger, J., Sauter, N. K., Bergmann, U., Yano, J., Yachandra, V. K. \& Bogan, M. J. (2012). Acta Cryst. D68, 1584-1587.

Soares, A. S., Mullen, J. D., Parekh, R. M., McCarthy, G. S., Roessler, C. G., Jackimowicz, R., Skinner, J. M., Orville, A. M., Allaire, M. \& Sweet, R. M. (2014). J. Synchrotron Rad. 21, 1231-1239.

Stellato, F., Oberthür, D., Liang, M., Bean, R., Gati, C., Yefanov, O., Barty, A., Burkhardt, A., Fischer, P., Galli, L., Kirian, R. A., Meyer, J., Panneerselvam, S., Yoon, C. H., Chervinskii, F., Speller, E., White, T. A., Betzel, C., Meents, A. \& Chapman, H. N. (2014). IUCrJ, 1, 204-212.

Vagin, A. \& Teplyakov, A. (2010). Acta Cryst. D66, 22-25.

Vonrhein, C., Flensburg, C., Keller, P., Sharff, A., Smart, O., Paciorek, W., Womack, T. \& Bricogne, G. (2011). Acta Cryst. D67, 293-302.

Weierstall, U., James, D., Wang, C., White, T. A., Wang, D., Liu, W., Spence, J. C., Bruce Doak, R., Nelson, G., Fromme, P., Fromme, R., Grotjohann, I., Kupitz, C., Zatsepin, N. A., Liu, H., Basu, S., 
Wacker, D., Won Han, G., Katritch, V., Boutet, S., Messerschmidt, M., Williams, G. J., Koglin, J. E., Marvin Seibert, M., Klinker, M., Gati, C., Shoeman, R. L., Barty, A., Chapman, H. N., Kirian, R. A., Beyerlein, K. R., Stevens, R. C., Li, D., Shah, S. T., Howe, N., Caffrey, M. \& Cherezov, V. (2014). Nat. Commun. 5, 3309.
Zander, U., Bourenkov, G., Popov, A. N., de Sanctis, D., Svensson, O., McCarthy, A. A., Round, E., Gordeliy, V., Mueller-Dieckmann, C. \& Leonard, G. A. (2015). Acta Cryst. D71, 2328-2343.

Zeldin, O. B., Gerstel, M. \& Garman, E. F. (2013). J. Appl. Cryst. 46, $1225-1230$ 